\title{
On the Fe abundance peak formation in cool-core clusters of galaxies: hints from cluster WARPJ1415.1+3612 at $z=1.03$
}

\author{
Sabrina De Grandi ${ }^{1}$, Joana S. Santos ${ }^{2}$, Mario Nonino ${ }^{3}$, Silvano Molendi ${ }^{4}$, Paolo Tozzi ${ }^{3}$, Mariachiara Rossetti ${ }^{5}$, \\ Alexander Fritz ${ }^{4}$, and Piero Rosati ${ }^{6}$
}

1 INAF, Osservatorio Astronomico di Brera, via E. Bianchi 46, 23807 Merate, Italy e-mail: sabrina.degrandi@brera.inaf.it

2 INAF, Osservatorio Astrofisico di Arcetri, Largo Enrico Fermi 5, 50125 Firenze, Italy e-mail: [jsantos;ptozzi]@arcetri.astro.it

3 INAF, Osservatorio Astronomico di Trieste, via G.B. Tiepolo 11, 34143 Trieste, Italy e-mail: nonino@oats.inaf.it

4 INAF - IASF, via Bassini 15, 20100 Milano, Italy e-mail: [silvano;afritz]@iasf-milano.inaf.it

5 Università di Milano, 20100 Milano, Italy e-mail: mariachiara.rossetti@unimi.it

${ }^{6}$ Università di Ferrara, Via Saragat 1, 44122 Ferrara, Italy e-mail: rosati@fe.infn.it

Received 2 April 2014 / Accepted 30 May 2014

ABSTRACT

\begin{abstract}
We present a detailed study of the iron content of the core of the high-redshift cluster WARPJ1415.1+3612 $(z=1.03)$. By comparing the central Fe mass excess observed in this system, $M_{\mathrm{Fe}}^{\text {exc }}=(1.67 \pm 0.40) \times 10^{9} M_{\odot}$, with those measured in local cool-core systems, we infer that the bulk of the mass excess was already in place at $z=1$, when the age of the Universe was about half of what it is today. Our measures point to an early and intense period of star formation most likely associated with the formation of the BCG. Indeed, in the case of the power-law delay time distribution with slope -1 , which reproduces the data of WARPJ1415.1+3612 best, half of the supernovae explode within $0.4 \mathrm{Gyr}$ of the formation of the BCG. Finally, while for local cool-core clusters the Fe distribution is broader than the near-infrared light distribution of the BCG, in WARPJ1415.1+3612 the two distributions are consistent, indicating that the process responsible for broadening the Fe distribution in local systems has not yet started in this distant cluster.
\end{abstract}

Key words. galaxies: clusters: intracluster medium - galaxies: elliptical and lenticular, cD - X-rays: galaxies: clusters

\section{Introduction}

The deep potential wells of clusters of galaxies are permeated by a hot ( $k T \sim 1-10 \mathrm{keV})$, optically thin and spatially diffused plasma, the intra-cluster medium (ICM), which is also enriched by heavy elements. It follows that observations in the X-ray band provide a powerful method for studying and classifying these systems. Galaxy clusters can be divided into two main classes, cool-core and non cool-core systems, on the basis of the thermodynamical and chemical properties of their central regions (i.e. within radii $\simeq 0.15 r_{500}$ ). Cool-core clusters show an almost regular, elliptical morphology on large scales (e.g. Nurgaliev et al. 2013), and their innermost region displays several characteristic properties: the surface brightness and the iron $(\mathrm{Fe})$ abundance are both strongly peaked at the centre (where central cooling times are significantly shorter than the Hubble time) (e.g. Santos et al. 2008; Sanderson \& Ponman 2010), the temperature decreases to about one third of the external value (e.g. Hudson et al. 2010; Panagoulia et al. 2014), and the central entropy is $\$ 30 \mathrm{keV} \mathrm{cm}^{2}$ (e.g. Cavagnolo et al. 2009; Pratt et al. 2010; McDonald et al. 2013). Conversely, non-cool-core clusters do not show prominent surface-brightness peaks and temperature drops in the cores, and very often, they feature the irregular $\mathrm{X}$-ray morphologies and galaxy distributions indicative of recent merging events.
The study of the spatial distribution of iron in the ICM provides important information that can be used to deepen our understanding of the chemical history and evolution of galaxy clusters in general and of cool-core clusters in particular. Outside the core, the Fe abundance of the ICM is on average one-fourth solar for both cool-core and non-cool-core clusters (De Grandi \& Molendi 2001, hereafter DM01; Leccardi et al. 2010; Matsushita 2011), and it shows only a moderate evolution up to a redshift of $\sim 1.3$ (Balestra et al. 2007; Maughan et al. 2008; Baldi et al. 2012).

The abundance excess found at the centre of cool core clusters is always associated with a giant, early-type galaxy, the brightest cluster galaxy (BCG), often showing a very extended diffuse stellar envelope. The favoured scenario for the formation of the BCGs is through mergers of galaxies falling in along primordial filaments early in the history of the Universe (e.g. Dubinski 1998; De Lucia \& Blaizot 2007; Whiley et al. 2008; Collins et al. 2009; Hung \& Ebeling 2012; Ruszkowski \& Springel 2009; Lin et al. 2013; Lidman et al. 2013). The presence of a BCG makes this galaxy the prime candidate for producing the peaked abundance profiles (e.g. Fukazawa et al. 2000; Böhringer et al. 2004; De Grandi et al. 2004; Leccardi \& Molendi 2008a). De Grandi et al. (2004) estimated that BCGs in low-redshift clusters (i.e. $z<0.1$ ) are able to produce the Fe mass observed in the cluster core during their star formation 
histories. Furthermore, the observations show that the stellar light profiles of the BCGs are less extended than the Fe abundance profiles, suggesting that there must be a mixing mechanism that moved the metals from their production sites (DM01; Churazov et al. 2003; Rebusco et al. 2005; Graham et al. 2006).

Very little is known about the formation time and subsequent evolution of these peaked Fe abundance profiles in coolcore clusters. Baldi et al. (2012) present the first attempt to determine, with XMM-Newton and spatially resolved data, the evolution of the abundance at different positions in the clusters and with redshift (up to $z \sim 1.4$ ) and find no evidence of any evolution in the Fe content in the cores, although with low statistical significance and without distinguishing cool cores from non-cool cores. The major sources of $\mathrm{Fe}$ in the Universe are type-Ia supernovae (SNIa), whereas other elements (alpha elements) are produced by core-collapsed SN (SNcc) or by a mix of the two SN types. A measure of the alpha/Fe ratios in the ICM gives a first-order approximation of the relative importance of the $\mathrm{SN}$ types to the enrichment (for a review see Werner et al. 2008; Borgani et al. 2008). Early claims of an increasing type-Ia supernovae (SNIa) contribution, with respect to the SN core-collapsed (SNcc) to the ICM enrichment towards cluster centres (Finoguenov et al. 2000; Rasmussen \& Ponman 2007), contributed to establishing a scenario where the Fe abundance in cores increased steadily from the time of formation to $z=0$ (e.g. Fukazawa et al. 2000; Böhringer et al. 2004). More recent observations from XMM-Newton, Chandra, and Suzaku (e.g. Tamura et al. 2004; Million et al. 2011; Sato et al. 2007; Matsushita et al. 2013), which found a constant ratio between $\mathrm{SN}$ types at any radius in cool-core clusters contradicting the early results, requires to reconsider this picture.

In this paper we provide a detailed investigation of iron content and spatial distribution in the X-ray-selected cluster WARPJ1415.1+3612 (hereafter WARPJ1415) at redshift $z=1.03$ (Perlman et al. 2002; Fritz et al. 2009), using a deep Chandra observation recently explored by Santos et al. (2012, hereafter S12). This cluster is a high-redshift, strong cool-core cluster (S12, Santos et al. 2010), which is strikingly similar in its thermodynamic characteristics to nearby cool-core clusters. In particular we study the connection between its Fe content and its central galaxy, which was observed with the Subaru and Spitzer telescopes. Our aim is to compare the properties of this distant cluster with those of local (i.e. $z<0.1$ ) cool-core systems to try to constrain the formation history of their iron peaks (DM01; De Grandi et al. 2004). We extended the analysis in DM01 with $X M M-N e w t o n$ data, selecting from the XMM-Newton public archive a small sample of nearby clusters for which we also have near-infrared (NIR) observations of the central BCGs, in order to compare their chemical properties with those of the distant cluster WARPJ1415.

The structure of this paper is as follows. In Sect. 2, we present the X-ray and NIR datasets and the data analysis procedures used to study WARPJ1415 and the nearby cool-core clusters. In Sect. 3, we measure the Fe content in WARPJ1415 and in the local clusters and compare them. We estimate the enrichment times and the supernovae rates needed to produce the observed Fe mass in WARPJ1415. Section 4 is devoted to comparing the BCG's star-light profiles and the Fe abundance distributions in the ICM in our distant and local clusters and to discussing the ensuing implications for the enrichment histories in cool-core cluster cores. Finally, we summarize our results in Sect. 5.

Throughout this paper we assume the concordance flat $\Lambda \mathrm{CDM}$ cosmology with $H_{0}=71 \mathrm{~km} \mathrm{~s}^{-1} \mathrm{Mpc}^{-1}, \Omega_{\mathrm{m}}=0.27$, and $\Omega_{\Lambda}=0.73$, that for WARPJ1415 at $z=1.03$ gives a scale of $8.099 \mathrm{kpc} /{ }^{\prime \prime}$. Iron abundances are measured relative to the solar photospheric values of Asplund et al. (2009), where the solar $\mathrm{Fe}$ abundance is $Z_{\mathrm{Fe}, \odot}=3.16 \times 10^{-5}$.

Quoted errors are at the $1 \sigma$ level, unless otherwise stated.

\section{Data and analysis}

\subsection{WARPJ1415}

\subsubsection{X-ray data}

The galaxy cluster WARPJ1415 was detected in the Wide Angle ROSAT Pointed Survey (Jones et al. 1998; Perlman et al. 2002) and then observed by Chandra ACIS-S (280 ks) and ACIS-I (90 ks). S12 performed a detailed spatially resolved spectral analysis using both Chandra datasets for a total of $370 \mathrm{ks}$ exposure time. Their analysis so far represents the most detailed $\mathrm{X}$-ray analysis of any cool-core cluster at such high redshift, $z \sim 1$. Details on the data reduction with the CIAO 4.3 software package with CALDB 4.4.5 (Fruscione et al. 2006) are given in Sect. 2 of S12. In S12 the authors extracted ACIS-I and ACIS-S spectra from nine rings at different radii up to $\sim 400 \mathrm{kpc}$ and performed the spectral analysis on the combined spectra (apart for the last ring, 300-400 kpc, where there was no useful signal in the ACIS-I data) by assuming a single-temperature model. The resulting projected and de-projected temperature and Fe abundance profiles are shown in their Fig. 2. (Details on spectral analysis and de-projection technique are in S12.)

The high X-ray count statistic of WARPJ1415 allows the most detailed analysis of the $\mathrm{Fe}$ abundance distribution in the core of a cluster at $z \sim 1$ to date. We performed a new spectral analysis with the XSPEC package (v12.6, Arnaud 1996) starting from the same spectra, response matrices, and the ancillary response matrices computed by $\mathrm{S} 12$, but using the apec spectral model instead of the mekal one. We decided to use apec because this model is continuously updated within XSPEC and we want to be consistent with the analysis of the XMM-Newton data of the local clusters (see Sect. 2.2.1). We first checked that our re-analysis agrees with those published in S12. To this aim we combined the ACIS-S and ACIS-I spectra and assumed a singletemperature apec model over the energy range $0.5-7.0 \mathrm{keV}$ and a fixed local absorption equal to the Galactic neutral hydrogen column density $\left(N_{\mathrm{H}}\right)$ measured at the cluster position (Kalberla et al. 2005) of $N_{\mathrm{H}}=1.05 \times 10^{20} \mathrm{~cm}^{-2}$. The gas temperature, metal abundance, and normalization were left unconstrained in the fitting procedure. The resulting projected and de-projected temperature and $\mathrm{Fe}$ abundance profiles are consistent within the uncertainties with those published in S12.

We tested whether the Fe abundance might be affected by biases induced by the presence of multi-phase gas in the cluster core. We do not expect the abundance measurement to be strongly affected by the Fe-bias (Buote 2000a,b) or by the inverse Fe-bias (Rasia et al. 2008; Simionescu et al. 2009; Gastaldello et al. 2010) since the measured emission-weighted temperature in the innermost bin is $>4 \mathrm{keV}$, and the $\mathrm{Fe} \mathrm{L}$-shell complex (energy at rest $\sim 0.7-1.5 \mathrm{keV}$ ) for this cluster at $z=1$ only marginally overlaps the energy range considered for the spectral analysis $(\sim 0.35-0.7 \mathrm{keV}$ observer frame for a $z=1$ cluster). We checked the stability of the Fe abundance measurement by fitting the combined ACIS-S (495 cts) and ACIS-I (94 cts) spectrum of the innermost bin, a circle with a $20 \mathrm{kpc}$ radius, with a two-temperature apec model, where the temperature of the first component is fixed and the temperature of the second component is left free to vary. The values assigned to the first 
Table 1. Best-fitting values for a 2-temperature apec model for the ACIS (S and I) spectrum of the innermost ring.

\begin{tabular}{lcccc}
\hline \hline$T 1$ fixed & $T 2$ free & $N 1 / N 2$ & $Z_{\mathrm{Fe}}$ & C-stat \\
\hline 0.3 & $4.94_{-0.56}^{+0.67}$ & 0.261 & $3.61_{-1.06}^{+1.59}$ & 199.82 \\
0.5 & $4.98_{-0.58}^{+0.73}$ & 0.094 & $3.69_{-1.09}^{+1.63}$ & 200.01 \\
0.8 & $4.95_{-0.60}^{+0.78}$ & 0.052 & $3.78_{-1.12}^{+1.67}$ & 200.56 \\
1.0 & $4.97_{-0.62}^{+0.81}$ & 0.050 & $3.78_{-1.12}^{+1.66}$ & 200.57 \\
1.5 & $5.11_{-0.74}^{+0.95}$ & 0.085 & $3.87_{-1.10}^{+1.70}$ & 200.72 \\
2.0 & $5.17_{-1.20}^{+2.18}$ & 0.147 & $4.05_{-1.65}^{+3.12}$ & 201.20 \\
2.5 & $4.91_{-0.97}^{+3.54}$ & 0.134 & $4.21_{-1.78}^{+3.96}$ & 201.65 \\
- & $4.44_{-0.36}^{+0.46}$ & - & $4.01_{-1.19}^{+0.99}$ & 202.00 \\
\hline
\end{tabular}

Notes. The columns are: (1) first component temperature that is fixed; (2) best-fit of the second component temperature; (3) ratio between the normalizations of the first and second components; (4) best fit of the Fe abundance in solar units; (5) Cash statistics of the best fit (always for 251 PHA bins). The last row shows the result for the single temperature fit of the spectrum.

temperature are reported in Table 1. As in S12 we use the Cash statistics, which is preferable for low $\mathrm{S} / \mathrm{N}$ spectra (Nousek \& Shue 1989). From the results of the best-fit shown in Table 1 it is clear that, if present, a second component provides a modest contribution to the total emission and that the value of the measured iron abundance is essentially unaffected by the multitemperature structure.

\subsubsection{Near-infrared data}

The BCG of WARPJ1415 is a large and massive elliptical galaxy with luminosity in the $B$ band of $L_{B}=3 \times 10^{11} L_{B, \odot}$. We retrieved the raw broadband images taken with Subaru/Suprime (BVRi' $z^{\prime}$ bands; Miyazaki et al. 2002) and Subaru/MOIRCS ( $J, K$ s bands; Suzuki et al. 2008), from the Subaru archive, SMOKA $^{1}$. MOIRCS provides wide-field imaging in a $4^{\prime} \times$ $7^{\prime}$ field of view covered by two $2048 \times 2048$ arrays with the spatial resolution of $0.117 \mathrm{arcsec} / \mathrm{pixel}$. The radial stellar profile in the NIR Ks band is extracted from MOIRCS data using the IRAF ELLIPSE routine, a procedure that measures best-fit isophotes from the data. The sky-background-subtracted profile shown in Fig. 1 is traced out to a radius of $\sim 80 \mathrm{kpc}$; however, beyond a radius of $\sim 30 \mathrm{kpc}$, the profile rapidly dilutes into the background.

Using our own multi-colour catalogue from broadband optical, infrared, and IRAC ${ }^{2}$ photometry, we estimated the stellar mass of the BCG by fitting the galaxy spectral energy distribution (SED) with LePhare (Arnouts \& Ilbert 2011) obtaining $M_{\star}=9.3 \times 10^{11} M_{\odot}$ for a Chabrier IMF (Chabrier 2003). With the SED fitting we measured an apparent, rest frame $K$ s magnitude of $18.69 \mathrm{AB}$. To estimate the $K$ s rest frame magnitude, we used all photometric points computed within $1.5^{\prime \prime}$ radius aperture, then corrected to $2.3^{\prime \prime}$ (18 kpc rest frame) aperture assuming no colour dependence, with respect to the $z$ band. This step

\footnotetext{
1 Based in part on data collected at Subaru Telescope and obtained from the SMOKA, Subaru-Mitaka-Okayama-Kiso Archive, which is operated by the Astronomy Data Centre, National Astronomical Observatory of Japan (Baba et al. 2002).

2 This work is based in part on observations made with the Spitzer Space Telescope obtained from the NASA/IPAC Infrared Science Archive, both of which are operated by the Jet Propulsion Laboratory, California Institute of Technology, under a contract with the National Aeronautics and Space Administration.
}

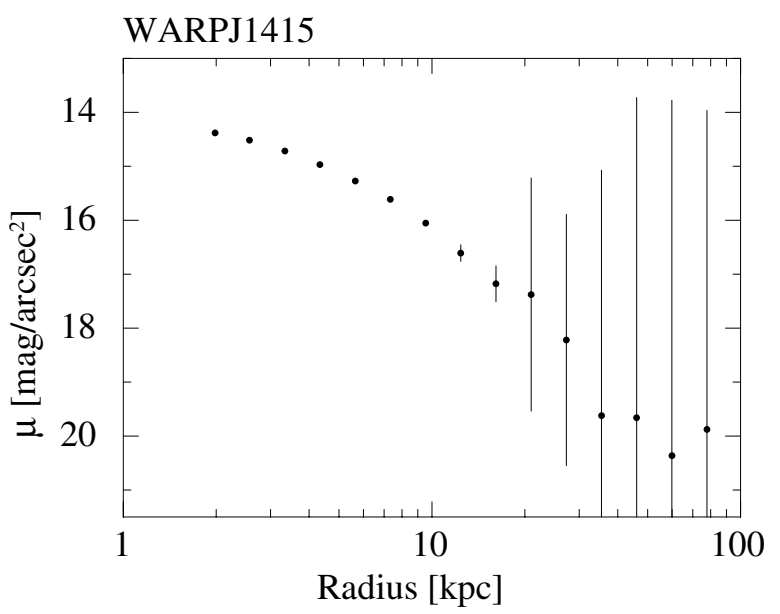

Fig. 1. Subaru/MOIRCS Ks surface brightness profile of the BCG in WARPJ1415.

Table 2. The local sample of galaxy clusters collected as a reference sample in this work.

\begin{tabular}{lccccc}
\hline \hline Name & $z$ & Obs. Id & $\begin{array}{c}t_{\mathrm{M} 1} \\
\mathrm{ks}\end{array}$ & $\begin{array}{c}t_{\mathrm{M} 2} \\
\mathrm{ks}\end{array}$ & $\begin{array}{c}t_{\mathrm{pn}} \\
\mathrm{ks}\end{array}$ \\
\hline A478 & 0.0329 & 0109880101 & 39.7 & 92.0 & 61.8 \\
A496 & 0.0329 & 0506260301 & 57.1 & 58.7 & 28.3 \\
& & 0506260401 & 53.0 & 47.2 & 30.8 \\
A2597 & 0.0852 & 0147330101 & 48.6 & 48.4 & 49.4 \\
A3526 & 0.0114 & 0406200101 & 104.8 & 98.5 & 91.9 \\
A4059 & 0.0475 & 0109950101 & 16.3 & 16.1 & 16.1 \\
& & 0109950201 & 22.8 & 22.9 & 19.3 \\
Hydra-A & 0.0539 & 0504260101 & 69.2 & 71.5 & 49.4 \\
\hline
\end{tabular}

Notes. The columns read as follows: (1) name, (2) redshift, (3) XMMNewton archival observation number and (4) effective exposure time for each EPIC detector. Multiple observations of the same cluster were summed up for the spectral analysis.

was adopted to avoid BCG flux contamination in IRAC bands from nearby sources. The estimated absolute $K$ s rest frame magnitude is $-24.73 \pm 0.20 \mathrm{AB}$.

\subsection{Local clusters of galaxies}

From its thermodynamical properties, the distant cluster WARPJ1415 is very similar to cool-core clusters in the nearby Universe. In this work, our aim is to study the chemical properties of WARPJ1415 by comparing it with those of local systems. To this purpose we selected, from the XMM-Newton public archive, six strong cool-core clusters at redshifts lower than 0.09 that have medium-high total masses (i.e. $M_{500} \gtrsim 3.5 \times 10^{14} M_{\odot}$, Chen et al. 2007), similar to the total mass of WARPJ1415 $\left(M_{500}=2.4 \times 10^{14} M_{\odot}, \mathrm{S} 12\right)$. In particular, we included in our sample A3526 (the Centaurus cluster) since its iron abundance profile is very peaked and reaches over-solar Fe abundance in the center, as in the case of WARPJ1415. We point out that the spatial resolution for these low-redshift clusters sample observed with XMM-Newton is very similar to the spatial resolution for a cluster observed with Chandra at $z \sim 1$. This follows from the fact that the ratio of the angular distances computed at $z=0.05$ and $z=1$ is similar to the ratio between the point spread functions of the XMM-Newton/EPIC and Chandra/ACIS instruments.

The local clusters are listed in Table 2. 
Table 3. $K$ s band observations parameters of the BCGs in the local clusters.

\begin{tabular}{llllc}
\hline \hline Name & $z p$ & $\mathrm{rms}_{\text {zp }}$ & $N$ & Seeing $\left({ }^{\prime \prime}\right)$ \\
\hline A478 & 22.301 & 0.132 & 50 & 0.81 \\
A496 & 22.316 & 0.016 & 30 & 0.63 \\
A2597 & 22.373 & 0.028 & 30 & 0.69 \\
A3526 & 22.417 & 0.154 & 16 & 0.98 \\
A4059 & 22.252 & 0.141 & 23 & 0.81 \\
Hydra-A & 22.269 & 0.057 & 38 & 0.75 \\
\hline
\end{tabular}

Notes. The columns are: (1) cluster name; (2) zero point in VEGA magnitudes (to convert from VEGA to $\mathrm{AB}$ magnitudes the correction is +1.86); (3) rms of the zero point for the night of measurement; (4) number of combined frames (each frame has $6 \mathrm{~s}$ exposure); and (5) seeing of image in arc seconds.

\subsubsection{X-ray data}

We analysed XMM-Newton archival data for the clusters in Table 2, following the data analysis described in details in our previous work De Grandi \& Molendi (2009). We reprocessed the observation data files (ODF) using the Science Analysis System (SAS) version 10.0.0. The effective exposure times of the observations obtained after soft proton cleaning are reported in Table 2. Using the soft-proton, cleaned event files we extracted spectra from annular regions centred on the cluster emission peaks. We subtracted the background using blank-sky fields for EPIC MOS and pn produced by Leccardi \& Molendi (2008b). All the spectral fits were performed with the XSPEC package using a one-temperature thermal model (apec) and a twotemperature thermal model (apec+apec). The Galactic hydrogen column is accounted for with a wabs absorption model component assuming a Galactic HI value by Kalberla et al. (2005). Redshifts, temperatures, iron abundances, and normalizations of the two models were left free to vary. Whenever the two-temperature fit provided significant improvements over the single-temperature model (using an F-test), we used the Fe abundance derived from the two-temperature model.

\subsubsection{Near-infrared data}

When deriving the NIR surface brightness profiles of the BCGs in the local cool-core clusters, we used Ks band data that were acquired using SofI (Moorwood et al. 1998) mounted on NTT telescope at the ESO/La Silla observatory. Since the targeted galaxies have an angular size ranging from 2 to 4 arcmin, the observations were done with the large field mode, corresponding to a field of view of $5 \times 5$ arcmin, with a pixel scale of $0.288 \mathrm{arsec} / \mathrm{pix}$.

We performed a standard data reduction of the raw frames and calibration standard star fields using IRAF procedures. The main steps of the processing are dark subtraction, flat-field correction, and sky subtraction. Given the large angular size of the galaxies, sky subtraction is achieved through an on-source/offsource imaging strategy, where one frame targeted the science object and the next observed a blank field. Photometric calibration standards (Persson et al. 1998) were acquired several times during the observation run. The scatter in the zero points ranges from 0.02 to $0.14 \mathrm{mag}$ (see Table 3 ).

The $K \mathrm{~s}$ band background subtracted surface brightness profiles for the BCGs in the local cluster sample are shown in
Table 4. ICM Fe mass computed within overdensity $\Delta=2500$, Fe mass excess, and magnitude within an aperture of $18 \mathrm{kpc}$ for the distant cluster WARPJ1415 and for the local XMM-Newton cluster sample.

\begin{tabular}{lcccc}
\hline \hline Name & $\begin{array}{c}r_{2500} \\
\mathrm{kpc}\end{array}$ & $\begin{array}{c}M_{\mathrm{Fe}, 2500} \\
10^{9} M_{\odot}\end{array}$ & $\begin{array}{c}M_{\mathrm{Fe}}^{\mathrm{exc}} \\
10^{9} M_{\odot}\end{array}$ & $\begin{array}{c}M_{\mathrm{K}} \\
\mathrm{mag}\end{array}$ \\
\hline WARPJ1415 & 317.0 & $7.57 \pm 1.26$ & $1.67 \pm 0.40$ & -24.73 \\
\hline A478 & 641.4 & $29.6 \pm 0.07$ & $7.56 \pm 0.64$ & -22.50 \\
A496 & 461.0 & $10.0 \pm 0.01$ & $3.99 \pm 0.13$ & -23.96 \\
A2597 & 465.2 & $9.10 \pm 0.10$ & $1.46 \pm 0.44$ & -23.47 \\
A3526 & 439.3 & $4.60 \pm 0.01$ & $0.82 \pm 0.01$ & -24.07 \\
A4059 & 473.2 & $10.4 \pm 0.05$ & $4.86 \pm 0.45$ & -24.09 \\
Hydra-A & 466.3 & $8.40 \pm 0.02$ & $1.30 \pm 0.14$ & -24.04 \\
\hline
\end{tabular}

Notes. (a) $M_{K}$ for the Centaurus cluster is from Hoffer et al. (2012).

Fig. 2. These $K$ s light profiles are integrated up to the background level so they encompass faint light contribution, such as haloes or intra-cluster light, up to measured radii. For A3526, the CCD frame border is at $1.4^{\prime}(\sim 17 \mathrm{kpc})$ from the centre of the $\mathrm{BCG}$, and therefore the decreasing of the profile at larger radii is a border effect. For this cluster we limited our analysis to radii up to $17 \mathrm{kpc}$. In all other cases, we verified that the CCD borders are well outside the range considered in these plots.

We performed a 1D fit of the BCG light profiles shown in Fig. 2 using a Sersic model and integrated the best-fit model up to a radius of $18 \mathrm{kpc}$ to obtain the aperture $K \mathrm{~s}$ magnitudes of the galaxies (except for A3526 where we used the magnitude estimated by Hoffer et al. 2012). The absolute $K$ s magnitudes (AB reference system) are reported in Table 4, and errors on the magnitudes are \pm 0.10 mag.

\subsection{A note on the near-infrared band}

To probe the mass distribution of the BCG in detail and to measure possible faint contribution from intra-cluster light, we have based our analysis on deep $K$ s band data rather than other filter passbands. Indeed, NIR luminosities and, in particular, $K$-band magnitudes, represent a more direct link with the stellar mass content of galaxies than the visual ones (Kauffmann \& Charlot 1998). The light in the visual bands is dominated by the brightest main-sequence stars, whose luminosity decreases as stellar populations age. In contrast, a sort of conspiracy in the post main sequence evolution of stars maintains the $\mathrm{K}$ band luminosity of a stellar population roughly constant when it ages (Charlot 1996). The $K$-band magnitudes vs. X-ray-derived physical quantities are expected to correlate with a smaller scatter with respect to the optical ones, and this will allow us to put stronger constraints on the link between the BCGs and the ICM properties.

The rest frame $K$ s band at $z \sim 1$ falls on the $4.5 \mu$ m channel of Spitzer/IRAC (Infrared Array Camera). However, IRAC does not have enough spatial resolution to effectively estimate surface brightness profile for the BCG of WARPJ1415 at $z=1.03$. We thus applied a $K$ correction to the measured Ks-band data, based on a fit to the galaxy SED, to compare the BCG magnitude with the local sample. We have not considered any size or luminosity evolution for the distant BCG.

The $K$ s profile of the distant BCG, shown in Fig.1, samples a slightly different rest frame with respect to the local BCGs, but still in the NIR band (i.e. in the $J$ band corresponding to $\sim 1.2 \mu \mathrm{m}$ ), where the contamination from stellar evolution of the brightest main-sequence stars is still negligible. 

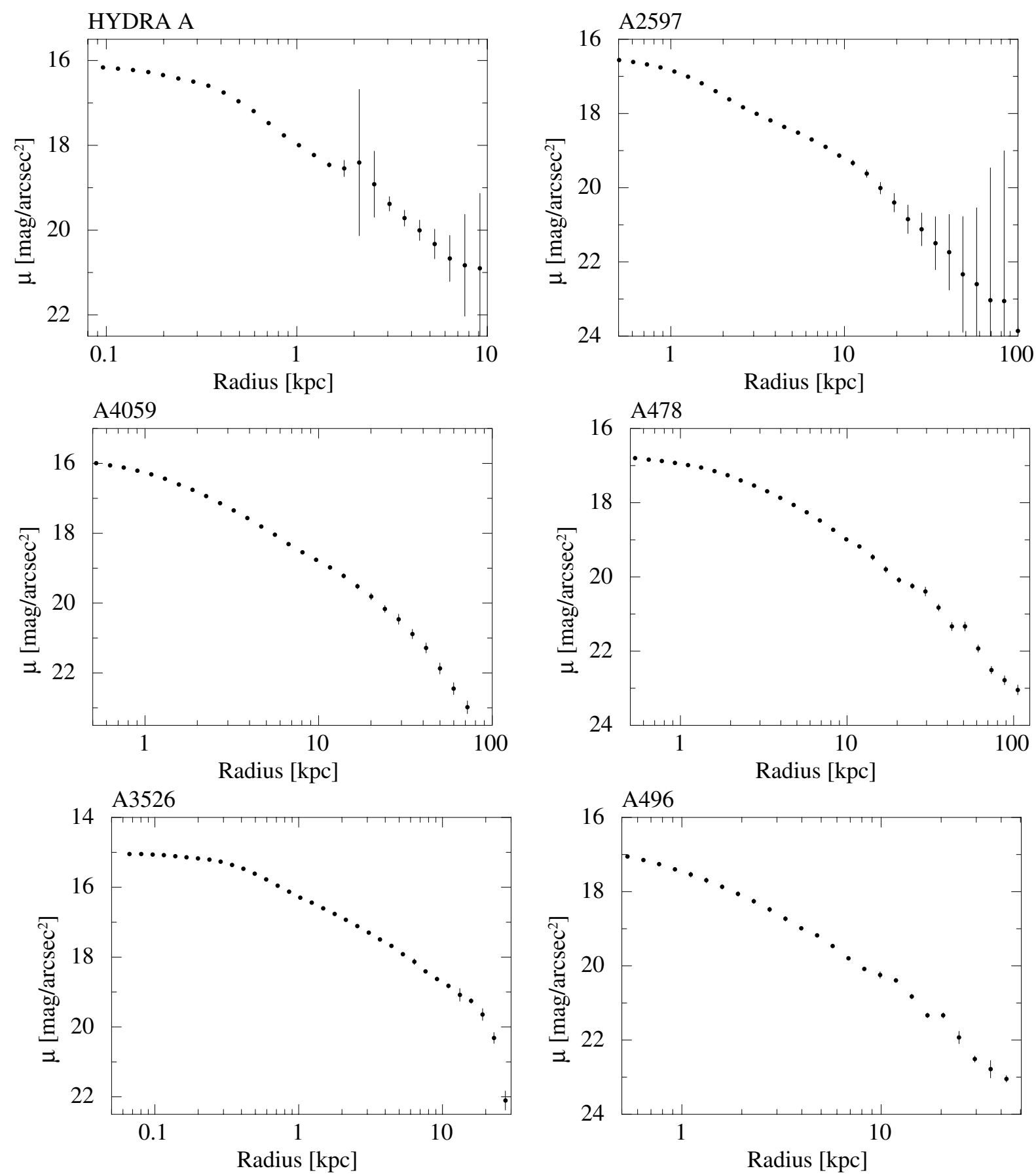

Fig. 2. $K$ s band surface brightness profiles of the BCGs in the local clusters listed in Table 2.

\section{Iron mass and iron mass excess}

\subsection{The iron mass}

The iron mass in the ICM (in solar units), enclosed within a sphere of radius $r^{\prime}$ from the cluster centre, is given by the following integral:

$M_{\mathrm{Fe}}\left(<r^{\prime}\right)=4 \pi A_{\mathrm{Fe}} m_{\mathrm{H}} \frac{Z_{\odot}}{M_{\odot}} \int_{0}^{r^{\prime}} Z_{\mathrm{Fe}}(r) n_{\mathrm{H}}(r) r^{2} \mathrm{~d} r$,

where $A_{\mathrm{Fe}}$ is the atomic weight of iron, $m_{\mathrm{H}}$ the atomic unit mass, and $Z_{\mathrm{Fe}}=n_{\mathrm{Fe}} / n_{\mathrm{H}}$ is the de-projected $\mathrm{Fe}$ abundance in solar units. ( $n_{\mathrm{Fe}}$ and $n_{\mathrm{H}}$ are the iron and hydrogen densities by number.) We estimate the iron mass in WARPJ1415 within $r_{2500}$ (i.e. $\$ 317_{-18}^{+22} \mathrm{kpc}$, see Table 1 in S12) linearly interpolating the cumulative iron mass profile computed with Eq. (1).

The errors on this mass are dominated by the systematics due to the large errors in the Fe abundance profile of WARPJ1415 for radii $\gtrsim 0.2 r_{200} \sim 180 \mathrm{kpc}(\mathrm{S} 12), Z_{\mathrm{Fe}}^{\text {out }}$. To estimate these systematic errors we compute the Fe mass with Eq. (1) twice, fixing $Z_{\mathrm{Fe}}^{\text {out }}$ to two extreme values: the best-fitting value of WARPJ1415 deprojected iron abundance profile at $r>0.2 r_{200}$ and the iron abundance outside the core measured in nearby cool-core clusters (DM01; Leccardi et al. 2010). The former value $(0.21 \pm 0.16)$ represents a lower limit for the $\mathrm{Fe}$ abundance outside the core in WARPJ1415, and it takes the conservative possibility of a positive evolution of $Z_{\mathrm{Fe}}^{\text {out }}$ from $z=1$ to $z=0$ into account. The latter value $(0.34 \pm 0.01$, from Leccardi et al. 2010, converted 
into Asplund et al. 2009 solar units) represents an upper limit to the Fe abundance in the cluster outskirts or, in other words, assumes that $Z_{\mathrm{Fe}}^{\text {out }}$ at redshift 1 is the same as the one at $z<0.3$ (Balestra et al. 2007; Leccardi et al. 2010). We finally compute the mean between these upper and lower Fe masses to derive the Fe mass within $r_{2500}: M_{\mathrm{Fe}, 2500}=(7.57 \pm 1.26) \times 10^{9} M_{\odot}$, with the systematic errors computed as half the difference between the two mass limits.

We de-project the XMM-Newton profiles of the local clusters in Table 2 following the method described in Ettori (2002) and Ghizzardi et al. (2004) and estimate the Fe masses of these clusters within $r_{2500}$ with Eq. (1). We use the relation $r_{2500} \sim 0.3 r_{200}$ (e.g. Ettori et al. 2010, see their Appendix) with $r_{200}$ taken from Reiprich \& Böhringer (2002). The Fe masses within $r_{2500}$ are given in Table 4. From this table and the results in DM01 (for clusters in common between the two samples we take the new $X M M$-Newton values), we find that the Fe mass distribution for cool-core clusters in the local Universe ranges between $\sim 0.4-6 \times$ $10^{10} M_{\odot}$ and that the Fe mass obtained for WARPJ1415 is consistent with the Fe masses measured in nearby clusters.

\subsection{The iron mass excess}

We use Eq. (1) to also measure the iron mass excess, $M_{\mathrm{Fe}}^{\mathrm{exc}}$, in WARPJ1415 and in the local clusters. This mass is computed by substituting in Eq. (1) $Z_{\mathrm{Fe}}$ with $Z_{\mathrm{Fe}}^{\text {exc }}$, which is defined as the iron abundance associated with the abundance peak in the cluster core after subtracting the average $\mathrm{Fe}$ abundance measured at radii larger than $0.2 r_{200}: Z_{\mathrm{Fe}}^{\text {exc }}(r)=Z_{\mathrm{Fe}}(r)-Z_{\mathrm{Fe}}^{\text {out }}$.

We compute the $\mathrm{Fe}$ mass excess and its errors for WARPJ1415 taking the two limits discussed previously for $Z_{\mathrm{Fe}}^{\text {out }}$ into account (i.e., 0.21 and 0.34$)$, obtaining $M_{\mathrm{Fe}}^{\text {exc }}=(1.67 \pm$ $0.40) \times 10^{9} M_{\odot}{ }^{3}$. This measure is consistent with the Fe mass excess distribution for nearby galaxy clusters: $\sim 1.0-10 \times 10^{9} M_{\odot}$ (see Table 4 and DM01). For WARPJ1415 we find a ratio $M_{\mathrm{Fe}}^{\mathrm{exc}} / M_{\mathrm{Fe}}$ of $0.22 \pm 0.06$, which again agrees within the uncertainties with the local cool-core clusters mean value, $0.20 \pm 0.05$.

By comparing the $K$ s absolute magnitude of the BCG in WARPJ1415 with those of the local sample shown in Table 4, we find that their values are comparable. This, and the similarity between the Fe mass excess in this and in local systems (see also De Grandi et al. 2004), strengthens our hypothesis that the iron present in the excess has also been produced in this distant cluster by the stars that form the BCG at $z=1$.

\subsection{Iron abundance excess formation time scale}

The high redshift of WARPJ1415 allows us to put some constraints on the formation time scale of the Fe peak. We use the simple formalization given by Böhringer et al. (2004),

$$
t_{\text {enrich }}=M_{\mathrm{Fe}}^{\mathrm{exc}}\left[L_{B}^{B C G}\left(\mathrm{SNR} 10^{-12} L_{B \odot}^{-1}\right) \eta_{\mathrm{Fe}}\right]^{-1}
$$

where $L_{B}^{\mathrm{BCG}}$ is the luminosity in the $B$-band of the BCG, SNR is the $\mathrm{SN}$ type Ia rate at the redshift of the cluster measured in $\mathrm{SNU}\left(1 \mathrm{SNU}=10^{-12} \mathrm{SNe} L_{B \odot}^{-1} \mathrm{yr}^{-1}\right)$, and $\eta_{\mathrm{Fe}}$ is the iron yield per $\mathrm{SN}$. In Eq. (2) the Fe mass excess is assumed to be entirely produced by Type Ia SNe neglecting additional minor contribution such as stellar mass loss or newly formed stars in the BCG. The

\footnotetext{
3 In agreement with $\mathrm{S} 12$, who find a Fe mass excess of $1.8_{-0.5}^{+0.7} \times$ $10^{9} M_{\odot}$, the small difference is due both to our slightly different spectral analysis and to the integration routine used to calculate the $\mathrm{Fe}$ mass excess.
}

rate of SNIa has been recently measured to be $0.50_{-0.23}^{+0.19} \mathrm{SNU}$ in galaxy clusters at $0.5<z<1.46$ (Barbary et al. 2012). This value suggests that there is little or no evolution in the cluster $\mathrm{SNe}$ rate from $z \sim 1$ to the present time. (For a compilation of recent cluster rate measurements see Dilday et al. 2010; Maoz $\&$ Mannucci 2012.) With a standard yield of $M_{\mathrm{Fe}}=0.7 M_{\odot}$ per SNIa (Iwamoto et al. 1999), we find $t_{\text {enrich }}=16.2 \mathrm{Gyr}$, which is significantly greater than the age of the Universe at the redshift of WARPJ1415 (i.e. $t_{H(z=1)}=5.9 \mathrm{Gyr}$ ). We conclude that current estimates of the $\mathrm{Fe}$ production rates at high redshift fail to account for the presence of the high Fe abundance peak at $z=1$ in WARPJ1415.

\subsection{Constraints on the $S N$ rate in BCGs at $z>1$}

As shown in the previous section, a constant SNR is inconsistent with our results, it is also inconsistent with other observational evidence and with theoretical work (e.g. Maoz \& Mannucci 2012). Maoz et al. (2010) argue that the SN delay time distribution (DTD) can be described as a power law with index close to -1 . To test that our data are consistent with such a model, we estimated the total number of SN that have exploded in the BCG by assuming a SNR of the form: SNR $=\operatorname{SNR}_{\mathrm{o}} \cdot\left(t / t_{\mathrm{o}}\right)^{-1}$, where $t_{\mathrm{O}}$ is the time that has passed between the formation of the BCG and our observation of WARPJ1415; $\mathrm{SNR}_{\mathrm{O}}$ is the $\mathrm{SN}$ rate at $t_{\mathrm{O}}$, which we derive by multiplying the stellar mass of our BCG, $M_{*}$, with the $\mathrm{SNR}$ per unit mass $(\mathrm{SNuM})$, i.e. $\mathrm{SNR}_{\mathrm{o}}=\mathrm{SNuM} \cdot M_{*}$, where we adopt the SNuM derived by Barbary et al. (2012) for highredshift red-sequence early type galaxies (see their Table 7); and $M_{*}$ is taken from Santos et al. (2012). This leads to a value of $\mathrm{SNR}_{\mathrm{o}}=0.474 \mathrm{yr}^{-1}$. The total number of SNIa, $\mathrm{SN}_{\text {tot }}$, is obtained by integrating the SNR from a minimum delay time, $t_{\mathrm{min}}$, to $t_{\mathrm{o}}$, which leads to $\mathrm{SN}_{\mathrm{tot}}=\mathrm{SNR}_{\mathrm{o}} t_{\mathrm{o}} \ln \left(t_{\mathrm{o}} / t_{\min }\right)$. Finally, the total mass of $\mathrm{Fe}$ produced is obtained by multiplying the total expected $\mathrm{SN}, \mathrm{SN}_{\text {tot }}$, by the yield per $\mathrm{SN}, \eta_{\mathrm{Fe}}$. Other authors (e.g. Greggio 2005) have proposed that at early times the SNR might fall off less rapidly, with a slope of $-1 / 2$. We have modelled the SNR in this scenario with a broken power-law model with index $-1 / 2$ from $t_{\min }$ to a break time, $t_{\mathrm{b}}$, and -1 from $t_{\mathrm{b}}$ to $t_{\mathrm{o}}$. In this case the total number of $\mathrm{SN}, \mathrm{SN}_{\text {tot }}$, turns out to be $\mathrm{SN}_{\text {tot }}=\mathrm{SNR}_{\mathrm{o}} t_{\mathrm{o}}\left\{2\left[1-\left(t_{\min } / t_{\mathrm{b}}\right)^{1 / 2}\right]+\ln \left(t_{\mathrm{o}} / t_{\mathrm{b}}\right)\right\}$.

Before comparing the Fe masses that can be estimated from different choices of the model parameters, we note that these predicted masses should be compared with the total Fe mass, which is given by the sum of the Fe excess mass observed in X-rays and the Fe mass locked up in stars in the BCG. The latter can be estimated from the total stellar mass assuming a typical metal abundance of $Z_{\mathrm{Fe}}=1.2$, (e.g. Maoz et al. 2010, and refs. therein). By performing this calculation, we find an Fe mass locked up in stars in the BCG of $2.1 \times 10^{9} M_{\odot}$ that, as expected, is close to the Fe excess and a total estimated Fe mass of $3.9 \times 10^{9} M_{\odot}$, which can now be compared with expectations from the different DTDs.

We then start by considering the case of a DTD shaped like a broken power law with $t_{\min }=0.04 \mathrm{Gyr}$ and $t_{\mathrm{b}}=0.4 \mathrm{Gyr}$ and consider the two cases of BCG formation at $z=2$ and $z=3$ corresponding to $t_{\mathrm{o}}$ of 2.2 and $3.6 \mathrm{Gyr}$, respectively. We find a total expected Fe mass of $1.8 \times 10^{9} M_{\odot}$ and $3.0 \times 10^{9} M_{\odot}$, respectively, but both these values fall short of the measured Fe mass in the case of a late BCG formation, $t_{\mathrm{o}}=2.2 \mathrm{Gyr}$, by more than a factor of 2 and in the case of an early formation, $t_{\mathrm{o}}=3.6$, by about $30 \%$. In the case of a power-law-shaped DTD with $t_{\min }=0.04 \mathrm{Gyr}$, we find a total expected Fe mass of $2.3 \times 10^{9} M_{\odot}$ for $t_{\mathrm{o}}=2.2 \mathrm{Gyr}$ and $3.8 \times 10^{9} M_{\odot}$ for $t_{\mathrm{o}}=3.6 \mathrm{Gyr}$, respectively. 
For the late BCG formation scenario, we are still below, by about a factor of 2 , the observed Fe mass, while for the early BCG formation scenario we are in good agreement.

Summarizing, our calculations suggest that a late BCG formation is unlikely, while an early BCG formation with a relatively flat SNR at early times, the broken power-law model, is below but possibly not inconsistent with the measured value. The best agreement is found with a combination of early BCG formation and steep SNR extending down to very short delay times, namely 0.04 Gyr.

At the end of this section we can draw some conclusions. The galaxy cluster WARPJ1415 at $z=1.03$, from its X-ray and NIR properties, is virtually indistinguishable from a strong coolcore cluster at $z \lesssim 0.1$. Indeed, its Fe mass and Fe mass excess, its luminosity, and the stellar mass of its central BCG all agree with what it is observed in local cool-core clusters.

The presence of a well-formed Fe peak at the centre of this distant cool-core cluster, all similar to the ones found in nearby clusters, places strong constraints on the formation time of the peak itself. As shown previously, the enrichment time of the $\mathrm{Fe}$ abundance peak is $\sim 4 \mathrm{Gyr}$, and to produce all the Fe in the central excess and in the stars of the BCG, we require SNIa rates that are much higher than those observed in both low-redshift or highredshift galaxy clusters.

The most straightforward implication of our results is that the bulk of the enrichment occurred at very early times, within a few $100 \mathrm{Myr}$ of the formation of the BCG, possibly during the BCG assembly process. Indeed, in the case of the power-law DTD, which best reproduces our data, half of the SN explode within 0.4 Gyr of the formation of the BCG. This is consistent with observational evidence showing that the star formation rate (SFR) during this time could have been extremely high. A good example is the so-called Spiderweb galaxy at $z=2$ with an observed SFR of $1390 \pm 150 M_{\odot} \mathrm{yr}^{-1}$ (Seymour et al. 2012), which converts into an equally huge SNR (e.g. Calura et al. 2007; Maoz et al. 2012). Other observations show that $\sim 50-60$ per cent of the iron released by SNIa explosions occurs within $\sim 1$ Gyr of a burst of star formation (e.g. Maoz et al. 2010, 2012, and references therein for the most recent measure of the delay-time distribution of SNe Ia as a function of time). Both observation (e.g. Daddi et al. 2004; Rosati et al. 2009; Collins et al. 2009; Stott et al. 2010) and theory (e.g. Dubinski 1998; Conroy et al. 2007a; De Lucia \& Blaizot 2007), point out that BCGs form at early times $(z>2)$ relatively quickly via hierarchical merging (or cannibalism) of small groups of galaxies falling along the filamentary large-scale structure prior to cluster virialization. The growth of the BCGs continue to be fed by mass flows up to $z=1.5-2$, when the stellar population of the BCG start to evolve mostly passively (e.g. Brodwin et al. 2013). Nearly all BCGs in the centres of local cool-core clusters still show little ongoing star formation, with SFRs that correlate with the ICM cooling rate (e.g. Rafferty et al. 2008; O’Dea et al. 2010; Donahue et al. 2010; McDonald et al. 2011); however, these rates contribute to a negligible Fe production. The SFR in WARPJ1415 of $\sim 2-8 M_{\odot} / \mathrm{yr}^{-1}$ (S12), as inferred from the [OII] emission line in the BCG spectrum, is comparable to typical SFRs measured in BCG in cool-core clusters at low redshift (i.e., $1-10 M_{\odot} / \mathrm{yr}^{-1}$ O'Dea et al. 2010), indicating that the major epoch of metal production in this cluster consistently happened at redshifts higher than 1 .

All these observations suggest that the bulk of the Fe abundance excess in WARPJ1415 was likely produced by the prompt release of the SNIa shortly after its BCG assembly at $z \gg 1$ and relatively short delay times.

\section{BCG stellar light and Fe abundance profiles}

In the previous section we found that the Fe mass excess in WARPJ1415 was most probably produced by the stars forming the BCG at redshifts $\gtrsim 1.5-2$. Now we want to compare the stellar light distribution of the BCG with the Fe abundance excess profile.

To this aim we compute the $\mathrm{Fe}$ abundance profile expected when the $\mathrm{Fe}$ distribution traces the light distribution of the BCG. Following DM01, we find that the projected abundance $Z_{\mathrm{Fe} \text {,proj }}\left(b_{\min }, b_{\mathrm{max}}\right)$ measured within a bin with bounding radii $b_{\min }, b_{\max }$ is related to the de-projected $\mathrm{Fe}$ abundance distribution, $Z_{\mathrm{Fe}}(r)$, by the following equation:

$Z_{\mathrm{Fe}, \mathrm{proj}}\left(b_{\min }, b_{\max }\right)=\frac{\int_{b_{\min }}^{b_{\max }} b \mathrm{~d} b \int_{b^{2}}^{\infty} \frac{n_{\mathrm{H}}^{2}(r) Z_{\mathrm{Fe}}(r)}{\sqrt{r^{2}-b^{2}}} \mathrm{~d} r^{2}}{\int_{b_{\min }}^{b_{\max }} b \mathrm{~d} b \int_{b^{2}}^{\infty} \frac{n_{\mathrm{H}}^{2}(r)}{\sqrt{r^{2}-b^{2}}} \mathrm{~d} r^{2}}$,

where $n_{\mathrm{H}}(r)$ is the hydrogen density (by number), and $Z_{\mathrm{Fe}}(r)=$ $n_{\mathrm{Fe}}(r) / n_{\mathrm{H}}(r)$ is the abundance excess profile with $n_{\mathrm{Fe}}(r)$ equal to the Fe density (by number). We assume that $n_{\mathrm{Fe}}(r)$ follows the stellar light distribution of the BCG, $l(r)$, i.e. $n_{\mathrm{Fe}}(r) \propto l(r)$, and then compute the projected $\mathrm{Fe}$ abundance profile expected, $Z_{\mathrm{Fe}, \text { proj }}$, under this hypothesis $\left(Z_{\mathrm{Fe}}(r) \propto l(r) / n_{\mathrm{H}}(r)\right)$ with Eq. (3). We, finally, compare this expected Fe excess profile with the observed one.

DM01 performed a similar comparison on a sample of local clusters observed with BeppoSAX, showing that the observed $\mathrm{Fe}$ abundance profile in the Perseus cluster was much broader than the predicted one. This result was later confirmed by several authors working on nearby clusters observed with the XMM-Newton satellite (e.g. Churazov et al. 2003; Rebusco et al. 2005; David \& Nulsen 2008). Under our assumption that the iron in the excess was formed by the stars in the BCG, this difference indicated that the Fe produced in the BCG spread out several tens of $\mathrm{kpc}$ from the place of origin.

We redid this exercise for the local cool-core clusters listed in Table 2 and for WARPJ1415 at $z=1$. The results are plotted in Fig. 3 for the local clusters and in Fig. 4 for WARPJ1415: in each panel the data points are the observed $\mathrm{Fe}$ abundance excess, whereas the continuous lines are the expected Fe abundance excess profiles. The profiles are arbitrarily normalized to the innermost bin because in this context, we are only interested in the comparison between the shapes of the profiles. From Fig. 3 it is clear that for all the local clusters, the observed profiles are substantially broader than the expected ones.

In Fig. 4, we plot two expected profiles for WARPJ1415 to include the uncertainties in the Ks band BCG's profile (see Fig. 1). These uncertainties have been included by computing the maximum and minimum expected $\mathrm{Fe}$ abundance excess profiles with Eq. (3) using the $K \mathrm{~s}$ band profile twice, the first time adding its $1 \sigma$ statistical errors to the $K$ s band profile and the second time, subtracting it (Fig. 4). Since the errors in the cumulative $K \mathrm{~s}$ band profiles are not independent, these systematic errors may be overestimated. We have also experimented with different spatial binnings of the X-ray Fe abundance profile and find consistent results. We conclude that, despite the larger scatter produced by the X-ray and NIR measurements in WARPJ1415, its observed $\mathrm{Fe}$ abundance excess profile is closer, or at most it differs marginally from the predicted one. This is at variance with what it is observed in low-redshift cool-core clusters, where the $\mathrm{Fe}$ abundance excess profiles are always significantly broader than the expected ones (Fig. 3). 
HYDRA A

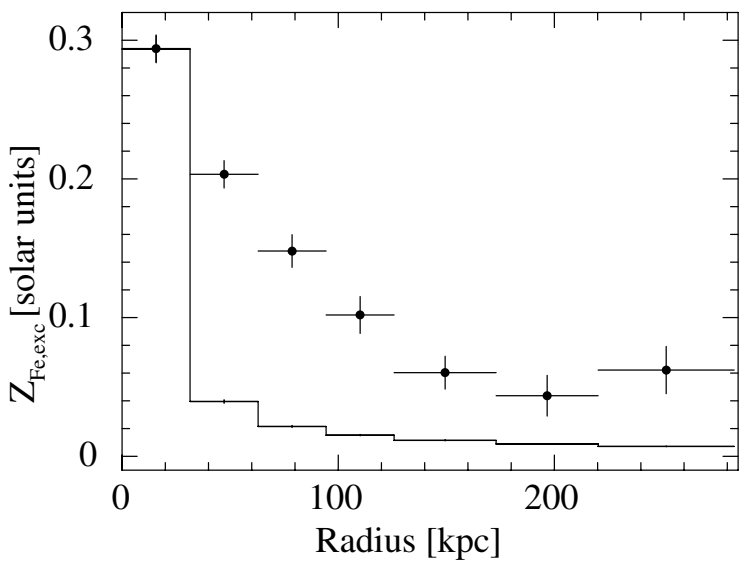

A4059
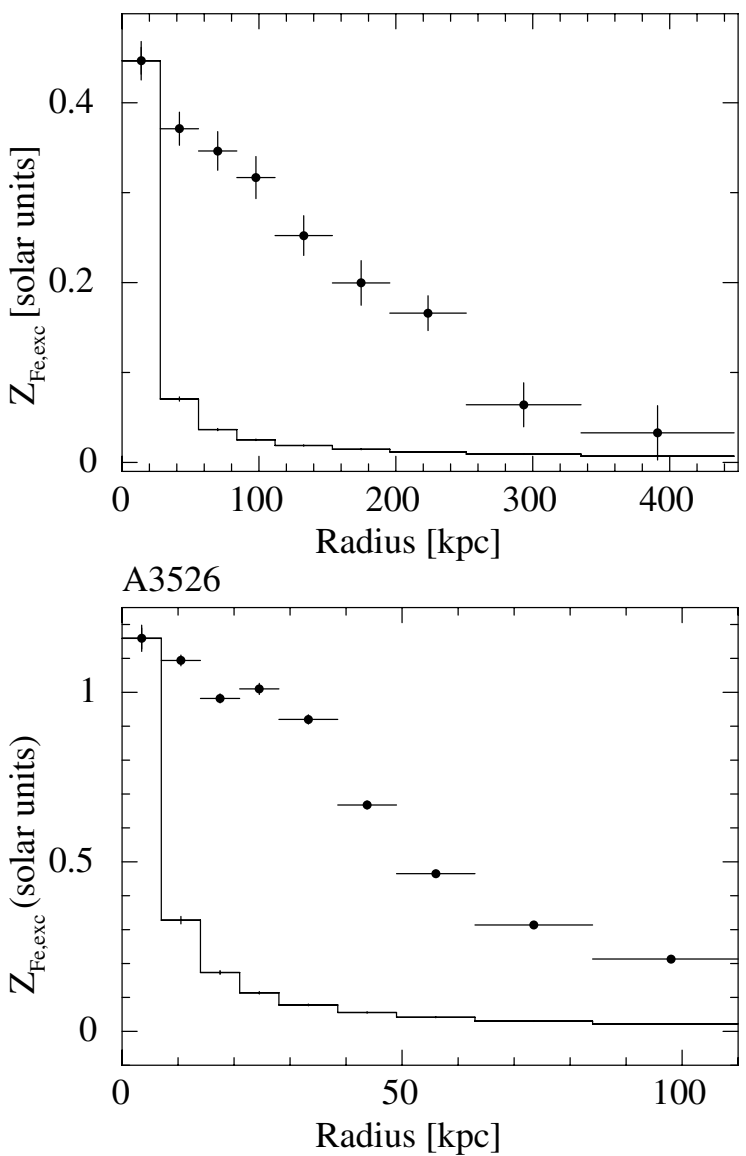

A2597

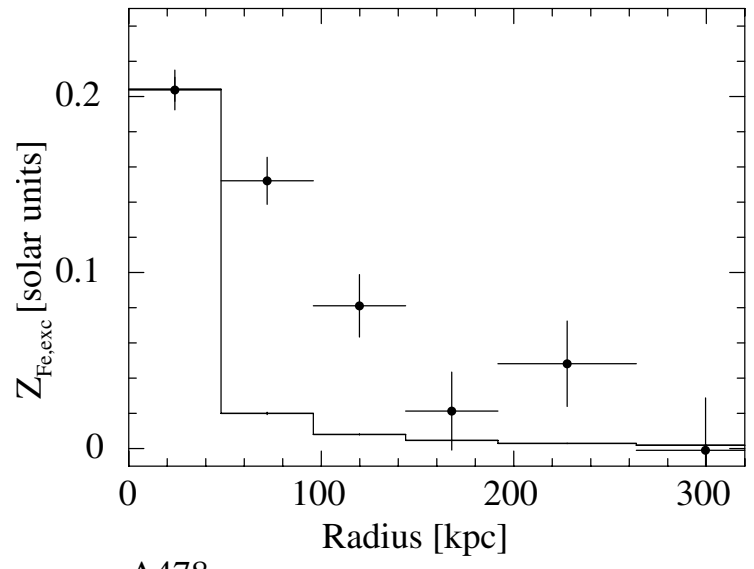

A478

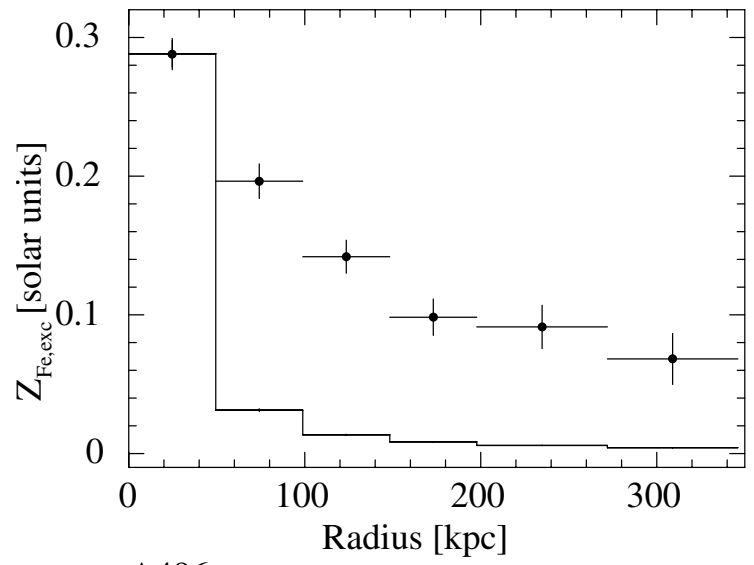

A496

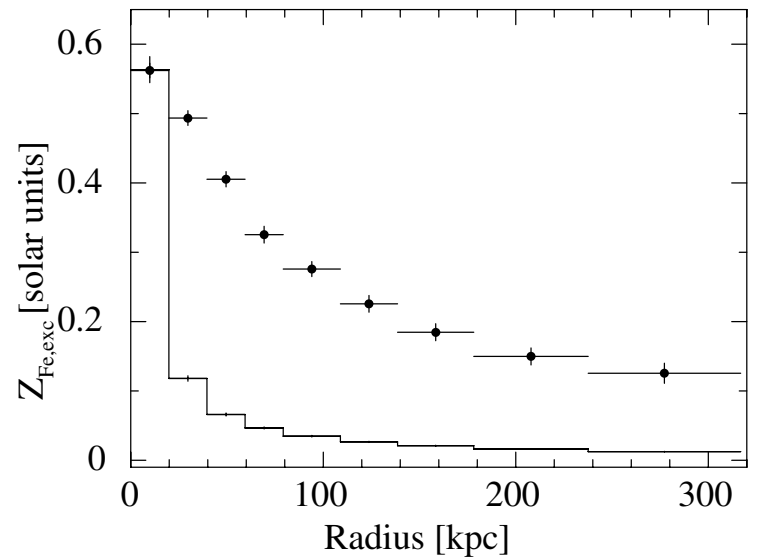

Fig. 3. Comparison between the observed (black dots) and expected (red lines) Fe abundance excess profiles for the galaxy clusters in the $X M M$ Newton local sample.

Under the reasonable assumption that WARPJ1415 will evolve into a present-day cool-core cluster, the smaller difference between the observed and expected profiles (Fig. 4) may indicate that the processes responsible for the broadening of the Fe excess profile in local systems have not yet had time to produce significant modifications in this distant cluster.

The time available for widening the Fe abundance peak excess is $\gtrsim 7.9 \mathrm{Gyr}$, i.e., the look-back time at $z=1.03$. On the basis of this observation alone, it is not possible to know whether there is a dominant mechanism that broadens the Fe profile or whether this widening is the result of the combined action of more mechanisms. Neither it is possible to hypothesize whether the Fe broadening is the result of many smaller events during a long period of time or if it is the result of a single event happening in a relatively short time period. What is apparent is that the time available to broaden the Fe peak is very long.

The mechanisms that could mix the ICM and produce the observed Fe profiles are several (see Schindler \& Diaferio 2008, and references therein). One possible mechanism is turbulent mixing of the ICM due to AGN activity (e.g. Rebusco et al. 2005; Simionescu et al. 2009, 2010; Gitti et al. 2011; O'Sullivan et al. 2011; McNamara et al. 2014). Kirkpatrick et al. (2011) found that clusters where the BCGs have experienced recent AGN activity (observed in the form of bright radio emission, cavities, and shock fronts embedded in the ICM) have heavy elements distributed anisotropically and aligned with the large-scale radio 


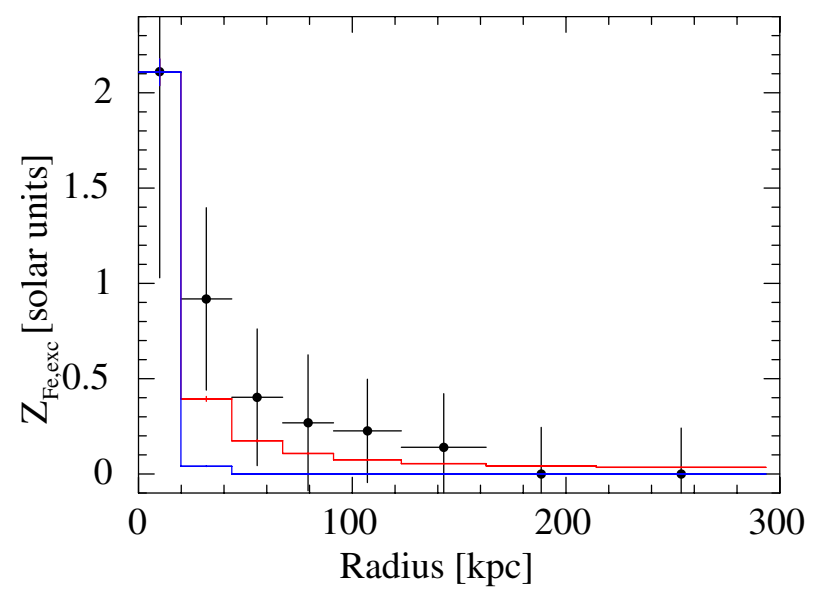

Fig. 4. Comparison between the observed (black dots) and expected (lines) Fe abundance excess profiles for WARPJ1415 at $z=1.03$. The red and blue lines are the expected profiles computed taking the errors in the observed BCG's Ks profile shown in Fig. 1 into account.

and cavity axes, indicating that they are being transported from the halo of the BCG into the ICM along the large-scale outflows driven by the radio jets. The amount of transported gas is substantial, and it is consistent with the results of simulations showing that AGN outflows are able to advect ambient, iron-rich material from the core to much larger radii of hundreds of $\mathrm{kpc}$ (Gaspari et al. 2011b,a; Planelles et al. 2014).

Another mechanism at work could be the ICM-sloshing induced by minor mergers in cool-core clusters (Ascasibar \& Markevitch 2006; Roediger et al. 2011), a phenomenon that is now more frequently discovered in nearby cool-core clusters (Simionescu et al. 2010; Ghizzardi et al. 2010). Indeed, in Ghizzardi et al. (2013) the observations of the central regions of A496 show that the gas-sloshing is able to shift the metals up to several tens of kpc out from the BCG (see also O'Sullivan et al. 2014).

Although still debated, the stellar mass of the BCGs appears to increase by a factor $\sim 2-3$ from redshift 1 to 0 (Lin et al. 2013; Laporte et al. 2013; Lidman et al. 2012, see however Collins et al. 2009; Stott et al. 2010). This mass accretion occurs through mergers due to fast and close encounters of small groups of galaxies onto the BCGs (e.g. Lidman et al. 2013; Tonini et al. 2012). The fate of the merging galaxy stars, which are collisionless, is to accrete onto the central giant galaxy increasing the stellar mass and the intra-cluster light surrounding such a galaxy (e.g. Conroy et al. 2007b; Murante et al. 2007; Henriques \& Thomas 2010). Indeed, once the streams of stars have been formed, they begin to decay since they are disrupted by the tidal field of the cluster within timescales that are $\simeq 1.5$ times their dynamical time in the cluster ( $t_{\text {dyn }} \lesssim 1$ Gyr, Rudick et al. 2009). Less obvious is the fate of the enriched ISM and of the infalling galaxies, which behave as a collisional fluid. This gas is probably tidally or ram-pressure stripped (Kapferer et al. 2007) from its disrupted galaxy and mixed with the ICM at a greater distance from the $\mathrm{BCG}$, with respect to the stars, in the end possibly contributing to the widening of the Fe peak between redshift 1 and 0 . The relative importance of this process should be investigated in depth both observationally and with numerical simulations (see Vazza et al. 2010).

\section{Summary}

Spatially resolved studies of the iron content in high-redshift clusters are very important because they provide much needed information on the enrichment process in these systems. Unfortunately, thus far, only a handful of high redshift cluster have been observed at the necessary sensitivity level, amongst them, the X-ray luminous cool-core cluster WARPJ1415 at $z=1.03$, which is the subject of this paper. Our main findings can be summarized as follows.

1. By comparing the central Fe mass excess from WARPJ1415 with those of local cool-core systems, we find that the Fe abundance excess produced by the BCG was already in place at $z=1$.

2. The large amount of metals observed at $z=1$ implies that the enrichment process must have occurred early on. More specifically, since $\mathrm{SN}$ rates at $z=1$ would imply enrichment timescales that are longer than the age of the Universe, our measures point to an early and intense period of star formation most likely associated with the formation of the BCG.

3. The Fe mass excess in WARPJ1415 agrees with expectations from the combination of early BCG formation $(z \sim 3)$ and steeply increasing SNR in the past extending down to very short, i.e. $0.04 \mathrm{Gyr}$, delay times.

4. While for local cool-core clusters the Fe distribution is broader than the NIR light distribution of the BCG, in WARPJ1415 the two distributions are consistent, indicating that the process responsible for broadening the Fe distribution in local systems has not yet started in this distant cluster.

Acknowledgements. The authors thank S. Borgani, S. Ghizzardi, and F. Mannucci for useful comments that helped to improve this work. S.D., P.T., S.M., M.R., and P.R. acknowledge the support by INAF PRIN 2012 (P.I. S. Molendi), A.F. acknowledges the support by INAF PRIN 2010 (P.I. L. Guzzo). J.S. has received funding from the European Programme FP7/2007-2013 under grant agreement 267251 "Astronomy Fellowships in Italy" (Astrofit). This research has made use of the NASA/IPAC Extragalactic Database (NED), which is operated by the Jet Propulsion Laboratory, California Institute of Technology, of the NASA's High Energy Astrophysics Science Archive Research Center (HEASARC), and, of the XMM-Newton archive.

\section{References}

Arnaud, K. A. 1996, in Astronomical Data Analysis Software and Systems V, eds. G. H. Jacoby, \& J. Barnes, ASP Conf. Ser., 101, 17

Arnouts, S., \& Ilbert, O. 2011, LePHARE: Photometric Analysis for Redshift Estimate, astrophysics Source Code Library

Ascasibar, Y., \& Markevitch, M. 2006, ApJ, 650, 102

Asplund, M., Grevesse, N., Sauval, A. J., \& Scott, P. 2009, ARA\&A, 47, 481

Baba, H., Yasuda, N., Ichikawa, S.-I., et al. 2002, in Astronomical Data Analysis Software and Systems XI, eds. D. A. Bohlender, D. Durand, \& T. H. Handley, ASP Conf. Ser., 281, 298

Baldi, A., Ettori, S., Molendi, S., et al. 2012, A\&A, 537, A142

Balestra, I., Tozzi, P., Ettori, S., et al. 2007, A\&A, 462, 429

Barbary, K., Aldering, G., Amanullah, R., et al. 2012, ApJ, 745, 32

Böhringer, H., Matsushita, K., Churazov, E., Finoguenov, A., \& Ikebe, Y. 2004, A\&A, 416, L21

Borgani, S., Fabjan, D., Tornatore, L., et al. 2008, Space Sci. Rev., 134, 379

Brodwin, M., Stanford, S. A., Gonzalez, A. H., et al. 2013, ApJ, 779, 138

Buote, D. A. 2000a, ApJ, 539, 172

Buote, D. A. 2000b, MNRAS, 311, 176

Calura, F., Matteucci, F., \& Tozzi, P. 2007, MNRAS, 378, L11

Cavagnolo, K. W., Donahue, M., Voit, G. M., \& Sun, M. 2009, ApJS, 182, 12

Chabrier, G. 2003, PASP, 115, 763

Charlot, S. 1996, in The Universe at High-z, Large-Scale Structure and the Cosmic Microwave Background, eds. E. Martinez-Gonzalez, \& J. L. Sanz, Lect. Notes Phys. (Berlin: Springer Verlag), 470, 53

Chen, Y., Reiprich, T. H., Böhringer, H., Ikebe, Y., \& Zhang, Y.-Y. 2007, A\&A, 466, 805

Churazov, E., Forman, W., Jones, C., \& Böhringer, H. 2003, ApJ, 590, 225

Collins, C. A., Stott, J. P., Hilton, M., et al. 2009, Nature, 458, 603

Conroy, C., Ho, S., \& White, M. 2007a, MNRAS, 379, 1491

Conroy, C., Wechsler, R. H., \& Kravtsov, A. V. 2007b, ApJ, 668, 826

Daddi, E., Cimatti, A., Renzini, A., et al. 2004, ApJ, 600, L127

David, L. P., \& Nulsen, P. E. J. 2008, ApJ, 689, 837 
De Grandi, S., \& Molendi, S. 2001, ApJ, 551, 153

De Grandi, S., \& Molendi, S. 2009, A\&A, 508, 565

De Grandi, S., Ettori, S., Longhetti, M., \& Molendi, S. 2004, A\&A, 419, 7

De Lucia, G., \& Blaizot, J. 2007, MNRAS, 375, 2

Dilday, B., Bassett, B., Becker, A., et al. 2010, ApJ, 715, 1021

Donahue, M., Bruch, S., Wang, E., et al. 2010, ApJ, 715, 881

Dubinski, J. 1998, ApJ, 502, 141

Ettori, S. 2002, MNRAS, 330, 971

Ettori, S., Gastaldello, F., Leccardi, A., et al. 2010, A\&A, 524, A68

Finoguenov, A., David, L. P., \& Ponman, T. J. 2000, ApJ, 544, 188

Fritz, A., Jørgensen, I., Schiavon, R. P., \& Chiboucas, K. 2009, Astron. Nachr. 330,931

Fruscione, A., McDowell, J. C., Allen, G. E., et al. 2006, in SPIE Conf. Ser., 6270,60

Fukazawa, Y., Makishima, K., Tamura, T., et al. 2000, MNRAS, 313, 21

Gaspari, M., Brighenti, F., D'Ercole, A., \& Melioli, C. 2011a, MNRAS, 415, 1549

Gaspari, M., Melioli, C., Brighenti, F., \& D’Ercole, A. 2011b, MNRAS, 411, 349

Gastaldello, F., Ettori, S., Balestra, I., et al. 2010, A\&A, 522, A34

Ghizzardi, S., Molendi, S., Pizzolato, F., \& De Grandi, S. 2004, ApJ, 609, 638

Ghizzardi, S., Rossetti, M., \& Molendi, S. 2010, A\&A, 516, A32

Ghizzardi, S., De Grandi, S., \& Molendi, S. 2013, Astron. Nachr., 334, 422

Gitti, M., Nulsen, P. E. J., David, L. P., McNamara, B. R., \& Wise, M. W. 2011, ApJ, 732, 13

Graham, J., Fabian, A. C., Sanders, J. S., \& Morris, R. G. 2006, MNRAS, 368, 1369

Greggio, L. 2005, A\&A, 441, 1055

Henriques, B. M. B., \& Thomas, P. A. 2010, MNRAS, 403, 768

Hoffer, A. S., Donahue, M., Hicks, A., \& Barthelemy, R. S. 2012, ApJS, 199, 23

Hudson, D. S., Mittal, R., Reiprich, T. H., et al. 2010, A\&A, 513, A37

Hung, C.-L., \& Ebeling, H. 2012, MNRAS, 421, 3229

Iwamoto, K., Brachwitz, F., Nomoto, K., et al. 1999, ApJS, 125, 439

Jones, L. R., Scharf, C., Ebeling, H., et al. 1998, ApJ, 495, 100

Kalberla, P. M. W., Burton, W. B., Hartmann, D., et al. 2005, A\&A, 440, 775

Kapferer, W., Kronberger, T., Weratschnig, J., et al. 2007, A\&A, 466, 813

Kauffmann, G., \& Charlot, S. 1998, MNRAS, 297, L23

Kirkpatrick, C. C., McNamara, B. R., \& Cavagnolo, K. W. 2011, ApJ, 731, L23

Laporte, C. F. P., White, S. D. M., Naab, T. \& Gao, L. 2013, MNRAS, 435, 901

Leccardi, A., \& Molendi, S. 2008a, A\&A, 487, 461

Leccardi, A., \& Molendi, S. 2008b, A\&A, 486, 359

Leccardi, A., Rossetti, M., \& Molendi, S. 2010, A\&A, 510, A82

Lidman, C., Suherli, J., Muzzin, A., et al. 2012, MNRAS, 427, 550

Lidman, C., Iacobuta, G., Bauer, A. E., et al. 2013, MNRAS, 433, 825

Lin, Y.-T., Brodwin, M., Gonzalez, A. H., et al. 2013, ApJ, 771, 61

Maoz, D., \& Mannucci, F. 2012, PASA, 29, 447

Maoz, D., Sharon, K., \& Gal-Yam, A. 2010, ApJ, 722, 1879

Maoz, D., Mannucci, F., \& Brandt, T. D. 2012, MNRAS, 426, 3282

Matsushita, K. 2011, A\&A, 527, A134

Matsushita, K., Sakuma, E., Sasaki, T., Sato, K., \& Simionescu, A. 2013, ApJ, 764, 147

Maughan, B. J., Jones, C., Forman, W., \& Van Speybroeck, L. 2008, ApJS, 174, 117
McDonald, M., Veilleux, S., Rupke, D. S. N., Mushotzky, R., \& Reynolds, C. 2011, ApJ, 734, 95

McDonald, M., Benson, B. A., Vikhlinin, A., et al. 2013, ApJ, 774, 23

McNamara, B. R., Russell, H. R., Nulsen, P. E. J., et al. 2014, ApJ, 785, 44

Million, E. T., Werner, N., Simionescu, A., \& Allen, S. W. 2011, MNRAS, 418, 2744

Miyazaki, S., Komiyama, Y., Sekiguchi, M., et al. 2002, PASJ, 54, 833

Moorwood, A., Cuby, J.-G., \& Lidman, C. 1998, The Messenger, 91, 9

Murante, G., Giovalli, M., Gerhard, O., et al. 2007, MNRAS, 377, 2

Nousek, J. A., \& Shue, D. R. 1989, ApJ, 342, 1207

Nurgaliev, D., McDonald, M., Benson, B. A., et al. 2013, ApJ, 779, 112

O'Dea, K. P., Quillen, A. C., O'Dea, C. P., et al. 2010, ApJ, 719, 1619

O'Sullivan, E., Giacintucci, S., David, L. P., Vrtilek, J. M., \& Raychaudhury, S. 2011, MNRAS, 411, 1833

O'Sullivan, E., David, L. P., \& Vrtilek, J. M. 2014, MNRAS, 437, 730

Panagoulia, E. K., Fabian, A. C., \& Sanders, J. S. 2014, MNRAS, 438, 2341

Perlman, E. S., Horner, D. J., Jones, L. R., et al. 2002, ApJS, 140, 265

Persson, S. E., Murphy, D. C., Krzeminski, W., Roth, M., \& Rieke, M. J. 1998, AJ, 116, 2475

Planelles, S., Borgani, S., Fabjan, D., et al. 2014, MNRAS, 438, 195

Pratt, G. W., Arnaud, M., Piffaretti, R., et al. 2010, A\&A, 511, A85

Rafferty, D. A., McNamara, B. R., \& Nulsen, P. E. J. 2008, ApJ, 687, 899

Rasia, E., Mazzotta, P., Bourdin, H., et al. 2008, ApJ, 674, 728

Rasmussen, J., \& Ponman, T. J. 2007, MNRAS, 380, 1554

Rebusco, P., Churazov, E., Böhringer, H., \& Forman, W. 2005, MNRAS, 359, 1041

Reiprich, T. H., \& Böhringer, H. 2002, ApJ, 567, 716

Roediger, E., Brüggen, M., Simionescu, A., et al. 2011, MNRAS, 413, 2057

Rosati, P., Tozzi, P., Gobat, R., et al. 2009, A\&A, 508, 583

Rudick, C. S., Mihos, J. C., Frey, L. H., \& McBride, C. K. 2009, ApJ, 699, 1518

Ruszkowski, M., \& Springel, V. 2009, ApJ, 696, 1094

Sanderson, A. J. R., \& Ponman, T. J. 2010, MNRAS, 402, 65

Santos, J. S., Rosati, P., Tozzi, P., et al. 2008, A\&A, 483, 35

Santos, J. S., Tozzi, P., Rosati, P., \& Böhringer, H. 2010, A\&A, 521, A64

Santos, J. S., Tozzi, P., Rosati, P., Nonino, M., \& Giovannini, G. 2012, A\&A, 539, A105

Sato, K., Tokoi, K., Matsushita, K., et al. 2007, ApJ, 667, L4

Schindler, S., \& Diaferio, A. 2008, Space Sci. Rev., 134, 363

Seymour, N., Altieri, B., De Breuck, C., et al. 2012, ApJ, 755, 146

Simionescu, A., Werner, N., Böhringer, H., et al. 2009, A\&A, 493, 409

Simionescu, A., Werner, N., Forman, W. R., et al. 2010, MNRAS, 405, 91

Stott, J. P., Collins, C. A., SahlŐn, M., et al. 2010, ApJ, 718, 23

Suzuki, R., Tokoku, C., Ichikawa, T., et al. 2008, PASJ, 60, 1347

Tamura, T., Kaastra, J. S., den Herder, J. W. A., Bleeker, J. A. M., \& Peterson, J. R. 2004, A\&A, 420, 135

Tonini, C., Bernyk, M., Croton, D., Maraston, C., \& Thomas, D. 2012, ApJ, 759, 43

Vazza, F., Gheller, C., \& Brunetti, G. 2010, A\&A, 513, A32

Werner, N., Durret, F., Ohashi, T., Schindler, S., \& Wiersma, R. P. C. 2008, Space Sci. Rev., 134, 337

Whiley, I. M., Aragón-Salamanca, A., De Lucia, G., et al. 2008, MNRAS, 387, 1253 